\title{
METODOLOGÍA PARA CALCULAR EL ÍNDICE DE POTENCIAL SOLAR INTRA-URBANO: INTEGRACIÓN DE VARIABLES MORFOLÓGICAS, TERMO-FÍSICAS, CLIMÁTICAS Y SOCIO-DEMOGRÁFICAS
}

\author{
P. J. Chévez ${ }^{1 *}$; G. Viegas ${ }^{1}$; I. Martini ${ }^{1}$; C. Discoli ${ }^{1}$
}

1 Instituto de Investigaciones y Políticas del Ambiente Construido. CONICET-UNLP, Argentina.

* che.pedro@hotmail.com

Artículo enviado el 29/06/2018 y aceptado el 13/07/2018

\section{RESUMEN}

La promoción de políticas energéticas orientadas a reducir la demanda y diversificar fuentes fósiles es una práctica cada vez más difundida a nivel global. En este contexto, la inserción de medidas de eficiencia energética y energías renovables en ámbitos urbanos constituye un aporte significativo a la temática. En relación a ello, generalmente, los estudios urbano-energéticos abordan a las ciudades como un elemento uniforme. Esto impide el direccionamiento de políticas específicas que se adecúen a las características del territorio. En consecuencia, este trabajo presenta una metodología para el análisis de la potencialidad de incorporación de sistemas de energía solar en distintos sectores urbanos, incluyendo variables morfológicas-urbanas, termo-físicas y de temperatura de cada sector; como así también el aporte de la dimensión socio-demográfica, que es fundamental para la aplicación de políticas energéticas. La metodología propuesta se sintetiza a continuación: (i) subdivisión y caracterización de la ciudad a partir de áreas homogéneas $(\mathrm{AH})$ urbanoenergéticas; (ii) selección y relevamiento morfológicourbano, termo-físico y climático de porciones urbanas representativas (mosaicos urbanos) en cada $\mathrm{AH}$; (iii) análisis del potencial solar intra-urbano en función de un índice multi-variable. Como conclusión se observan diferencias sustanciales entre las $\mathrm{AH}$, lo que conlleva al diseño de medidas específicas para cada sector.

PALABRAS CLAVE: Energía solar, Mosaico Urbano, Aspectos socio-demográficos.

\section{METHODOLOGY TO CALCULATE A INTRA-URBAN SOLAR POTENTIAL INDEX: INTEGRATION OF MORPHOLOGICAL, THERMO- PHYSICAL, CLIMATE AND SOCIO-DEMOGRAPHIC VARIABLES}

\begin{abstract}
The promotion of policies aimed at reducing energy demand and diversifying fossil fuels is an increasingly widespread practice worldwide. In this context, the insertion of energy efficiency measures and renewable energies in urban areas constitutes a significant contribution to the issue. In relation to this, generally, urban-energy studies approach cities as a uniform element. This impedes the promotion of specific policies designed for the particular characteristics of the territory. Consequently, this work presents a methodology for the analysis of the potential incorporation of solar energy systems in different urban sectors, including morphological-urban, thermo-physical and temperature
\end{abstract}

variables of each sector; as well as the contribution of the socio-demographic dimension, which is fundamental for the application of energy policies. The proposed methodology is synthesized as follows: (i) subdivision and characterization of the city from urban-energy homogenous areas (HA); (ii) selection and analysis of morphological-urban, thermo-physical and climatic variables of representative urban portions (urban mosaics) in each $\mathrm{AH}$; (iii) analysis of intra-urban solar potential based on a multi-variable index. In conclusion, substantial differences are observed between AHs, which leads to the design of specific measures for each sector.

KEYWORDS: Solar energy, Urban Mosaics, Socio-demographic aspects. 


\section{INTRODUCCIÓN}

En los últimos años el contexto energético mundial ha introducido el debate en la agenda política de la mayoría de los países acerca de la sustentabilidad, el uso eficiente de la energía, la utilización de energías renovables y la sustitución de combustibles fósiles. El crecimiento económico, desigual e intermitente, desarrollado desde mediados del siglo XX hasta la actualidad generó un consecuente incremento en la demanda energética, la cual es mayormente de origen convencional y de características extractivas. Se produjo, entonces, una fuerte dependencia hacia los combustibles fósiles; lo cual, en la actualidad, genera inconvenientes asociados a la volatilidad de sus precios, consecuentes impactos sobre las economías y tensiones geopolíticas permanentes por el control de los recursos. A su vez, no existen certezas sobre los niveles futuros de reservas de las principales fuentes como el petróleo y el gas. Y, por su parte, existe un consenso generalizado en señalar la necesidad de reducir sus niveles de consumo, ya que se han acentuado los efectos del cambio climático producto de las emisiones de gases de efecto invernadero; las cuales, en gran medida, son generadas por la utilización intensiva de fuentes fósiles.

Focalizando en el consumo de energía del sector residencial, el cual principalmente se localiza en ciudades, es posible observar que a nivel mundial este sector demanda cerca del $23 \%$ del total (IEA, 2015). Por su parte, el consumo energético residencial en Argentina alcanza el $27 \%$ del total, lo que lleva a situaciones de competencia con otros sectores, en particular con el industrial que tiene una participación del 22,5\% (MINEM, 2017). A su vez, aproximadamente un 50\% de las viviendas a nivel mundial y un $90 \%$ de las viviendas argentinas están localizadas en áreas urbanas (ONU, 2014; INDEC, 2016). Es por ello que se entiende que es fundamental focalizar los esfuerzos en reducir la demanda energética en las ciudades, a partir del mejoramiento de la eficiencia energética y de la incorporación de energías renovables, donde el sector residencial tiene un peso muy significativo. Esto posibilitará reducir considerablemente la demanda total y, por ende, minimizar las problemáticas energéticas, que en el caso argentino están asociadas a cortes de suministro, falta de potencia eléctrica en picos de demanda; importación de combustibles; y otras.

En relación a ello, a nivel mundial se pueden encontrar algunos ejemplos de acciones concretas donde se ha tomado como eje central a la ciudad como espacio para la incorporación masiva de energías renovables, la promoción de medidas de eficiencia energética para edificios nuevos/existentes y el incentivo de otro tipo de acciones (WEC, 2016). Sin embargo, su propagación aún es incipiente. En este sentido, los estudios de diagnóstico urbano-energéticos se posicionan como instrumentos de vital importancia para la evaluación y formulación de políticas energéticas, tanto para la demanda como para la oferta, siendo posible gestionar desde una óptica integrada las interacciones entre los principales vectores energéticos y los sectores que estructuran este consumo. Además, en general, los estudios que elaboran diagnósticos urbanos de energía abordan el área de estudio como un elemento uniforme sin considerar la diversidad intra-urbana. Por su parte, tampoco abundan los estudios que incluyan múltiples aspectos que relacionen la reducción de la demanda de energía para los diferentes sectores de un área de estudio, aplicando simultáneamente medidas de eficiencia energética (envolvente edilicia, sustitución de equipamiento domiciliario, etc.) y de inserción de energías renovables (activas y pasivas), y que además contemplen un enfoque de largo plazo.

La incorporación de alguno de los aspectos previamente mencionados se observan en Viegas (2010) o Amado et al. (2016), los cuales desarrollan metodologías para el análisis de mejoras de la envolvente de los edificios y el potencial solar en diferentes sectores urbanos, aunque carecen de una proyección temporal. En este sentido, existen experiencias que abordan la incorporación de estrategias energéticas en ámbitos urbanos con un enfoque de largo plazo, aplicando la técnica de escenarios, pero que utilizan como unidad de análisis a las tipologías de vivienda para ensayar medidas de mejoramiento energético, lo cual impide la interacción con variables territoriales 
(Drouilles et al., 2017; Ghiassi \& Mahdavi, 2017). Por otra parte, existen estudios que abordan los usos energéticos de los distintos sectores urbanos y analizan su demanda futura de forma global, sin posibilidades de ensayar medidas energéticas de forma precisa (Howard et al., 2012). También se encuentran trabajos donde se analiza el impacto de largo plazo de medidas específicas como la integración de un sistema de calefacción distrital o medidas de mejoramiento de la envolvente edilicia en sectores urbanos reducidos, lo cual impide identificar las diferencias intra-urbanas del caso de estudio (Girardin et al., 2010; Abdurafikov et al., 2017). A su vez, se encuentran trabajos de escala urbana que contribuyen directamente a los planificadores y tomadores de decisiones, donde se evalúan energéticamente las futuras expansiones urbanas, aunque su objetivo no es el ensayo de medidas de mejoramiento (Sveinbjörnsson et al., 2017; Mörtberg et al., 2016). Como se puede observar, los estudios urbano-energéticos no suelen abordar simultáneamente el problema con enfoques de largo plazo y aproximaciones intra-urbanas, ni tampoco integran en sus resultados otras variables relacionadas con las condiciones socio-demográficas de la población de la ciudad.

Con respecto a la determinación del potencial solar urbano e incorporación de sistemas no convencionales, se identifican trabajos con muy diversas técnicas de relevamiento. En este sentido, el trabajo de Gasdsen et al. (2003) parte de tipologías de vivienda representativas de la ciudad de Leicester (Reino Unido), sobre las cuales se ensaya la aplicación de sistemas solares pasivos, como así también sistemas de agua caliente sanitaria y fotovoltaicos bajo un entorno GIS. Una metodología similar se presenta en Wegertseder et al. (2016), donde se evalúa el potencial solar de sectores urbanos a partir de tipologías representativas, analizando el aporte de las tecnologías solares sobre el consumo energético diario y sobre las redes de distribución eléctricas, utilizando como caso de estudio a la ciudad chilena de Concepción. En Compagnon (2004) se presenta la simulación de un sector urbano, a partir de su modelado en tres dimensiones, en el cual se evalúa el potencial solar de fachadas y techos. Por su parte, el proyecto POLIS (2012) -Identification and mobilisation of solar potentials via local strategies- relevó el potencial solar de seis ciudades europeas con el objetivo de promover el uso de energía solar a escala urbana, generar insumos para la implementación de políticas y, fundamentalmente, ahorrar energía. Para dicho relevamiento se empleó la tecnología LiDAR, que permite crear un modelo digital de un sector urbano y, a partir de éste, obtener la potencialidad de las diferentes superficies que posee dicho sector. La tecnología LiDAR también se utiliza en otros trabajos para el relevamiento del potencial solar, como por ejemplo el de Brito et al. (2017), aplicado sobre la ciudad de Lisboa. También se han desarrollado trabajos a partir de modelos urbanos tridimensionales construidos en entorno CAD, como es el caso de Eicker et al. (2014), donde se evalúa el potencial solar y el reciclado de la envolvente edilicia de algunos sectores de la ciudad. En base estos antecedentes, es posible detectar que lo trabajos mayormente analizan el potencial solar en base a las propiedades físicas o morfológicas del sitio, sin considerar otro tipo de variables que puedan condicionar la incorporación de tecnologías solares. La identificación de las condiciones socio-demográficas o económicas, inciden directamente en la viabilidad de aplicación de los sistemas solares pasivos de calefacción, agua caliente sanitaria o generación eléctrica fotovoltaica, ya que permiten el direccionamiento de programas específicos que tengan en cuenta las condicionantes de cada sector urbano, lo cual evitaría el lanzamiento de programas genéricos que consideren al caso de estudio como un único elemento sin diferencias internas particulares.

En función de la identificación de la problemática y de la revisión de literatura, a continuación se exponen los objetivos y alcances del trabajo.

\section{OBJETIVOS Y ALCANCES DEL TRABAJO}

El presente trabajo plantea identificar el potencial para la incorporación de sistemas de energía solar en distintos sectores urbanos a partir de la integración de aspectos socio-demográficos, termo-físicos, morfológicos y climáticos. Asimismo, forma parte de un proyecto de investigación 
más amplio, el cual intenta abordar las vacancias señaladas en la revisión de literatura relacionadas con el análisis del impacto de diferentes medidas de mejoramiento energético tales como el recambio de equipamiento, mejoramiento de envolventes e incorporación de energías renovables sobre distintas áreas urbanas simulando los efectos de su implementación a través de escenarios en un horizonte temporal de largo plazo (Martini, 2016). Es por ello que en esta instancia se presenta pormenorizadamente la metodología desarrollada para la identificación y el análisis del potencial solar intra-urbano.

Para su desarrollo se plantea: (i) la delimitación de áreas homogéneas (AH) urbanoenergéticas, su caracterización socio-demográfica y la identificación de los niveles de consolidación urbana; (ii) el estudio de un mosaico urbano (MU) representativo de cada área, los cuales permiten relevar características morfológicas-urbanas, termo-físicas y climáticas. En base a dichos relevamientos, es posible (iii) calcular un índice de potencial de incorporación de sistemas de energía solar a los diferentes MUs, considerando simultáneamente variables de forma urbana y socio-demográficas, lo cual permite identificar las oportunidades y las barreras que presenta cada sector para la implementación de sistemas solares.

De esta manera, es posible contar con mayores herramientas que contribuyan a orientar de una manera más eficiente los recursos. A su vez, el potencial solar obtenido para cada área homogénea podrá ser utilizado, junto con otro tipo de medidas, para construir escenarios urbanoenergéticos en futuras publicaciones.

Por su parte, para la aplicación de la metodología se utiliza como caso de estudio a la ciudad de La Plata (Buenos Aires, Argentina), cuyas principales características se exponen a continuación.

\section{DESCRIPCIÓN DEL CASO DE ESTUDIO}

La ciudad de La Plata es la capital de la provincia de Buenos Aires (Argentina), se ubica al noreste de la misma, en las coordenadas $34^{\circ} 55^{\prime} \mathrm{S}$ y $57^{\circ} 17^{\prime} \mathrm{O}$. Se encuentra a $56 \mathrm{~km}$ al sudeste de la Ciudad Autónoma de Buenos Aires y a unos $10 \mathrm{~km}$ al sudoeste de la costa del Río de la Plata. La ciudad contaba para el año 2014 con una población estimada de unos 631.831 habitantes. Por su parte, la ciudad presenta ciertas características distintivas, principalmente asociadas al trazado de su casco urbano que fue proyectado desde cero para la fundación de la ciudad en 1882. El plano original se basó en la cuadrícula clásica utilizada en la mayoría de las ciudades bonaerenses, presente desde las Leyes de Indias, como así también en los preceptos higienistas que comenzaban a difundirse. El resultado final fue una cuadrícula de $5 \mathrm{~km}$ de lado, con manzanas de $120 \mathrm{~m}$ x $120 \mathrm{~m}$ (orientadas a medio rumbo), con dos bulevares centrales que dividen la ciudad en el eje NE-SO (eje cívico) y un sistema de diagonales, avenidas y plazas, superpuesto a la grilla ortogonal. Por su parte, el ancho de las avenidas quedó establecido en $30 \mathrm{~m}$ y el de las calles en $18 \mathrm{~m}$, atendiendo a generar fluidez en el tránsito y en la circulación del aire. Este último aspecto, ligado a las ideas higienistas. En este sentido, las manzanas adyacentes al eje cívico fueron proyectadas con una medida de 60 x $120 \mathrm{~m}$, las cuales incrementan su dimensión de a $10 \mathrm{~m}$ hasta alcanzar los $120 \mathrm{~m}$ de lado (Morosi, 1999). Posteriormente, los distintos códigos urbanos de la ciudad intentaron mantener estos preceptos regulando el crecimiento urbano en altura de acuerdo a cada zona. Esta situación hace de la ciudad un interesante caso de análisis para conocer su potencial de reducción de su consumo de energía e implementar tecnologías solares. El trazado original se puede observar en la Figura 1, el cual se encuentra señalado como 'Casco Urbano', y es el sector de mayor consolidación de la ciudad, a su vez, la ciudad ha sobrepasado los límites planteados inicialmente y se ha expandido de forma diferenciada en el territorio, pero en general respondiendo a un crecimiento de baja densidad principalmente sobre las grandes vías de comunicación. 


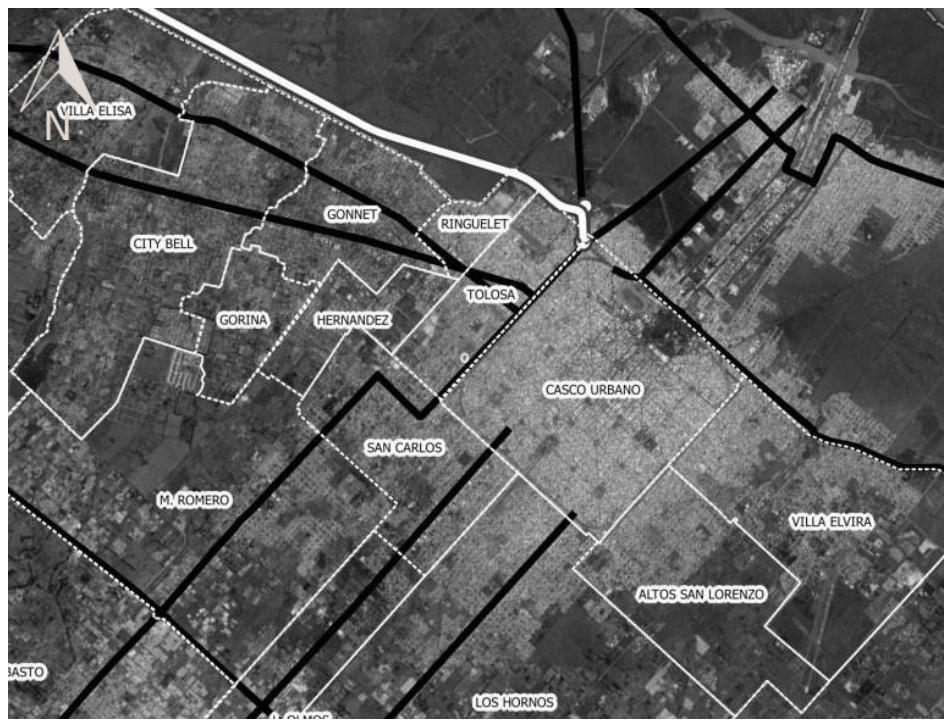

Figura 1 - Imagen aérea del caso de estudio (ciudad de La Plata). Escala: 1:250.000.

Fuente: Google Earth. Acceso el 1ero diciembre 2017.

\section{MÉTODOS: UTILIZACIÓN DE ÁREAS HOMOGÉNEAS Y MOSAICOS URBANOS PARA LA IDENTIFICACIÓN DEL POTENCIAL SOLAR DE DISTINTOS SECTORES URBANOS}

Tal como se describió en los objetivos y alcances, la metodología propuesta consta de tres etapas: (i) subdivisión y caracterización de la ciudad a partir de áreas homogéneas (AH); (ii) selección y relevamiento de mosaicos urbanos; y (iii) análisis del potencial solar intra-urbano en función de un índice multi-variable. Cada una de ellas se desarrolla a continuación.

\subsection{Métodos para la delimitación y caracterización de áreas homogéneas a partir de variables urbano-energéticas}

Las áreas homogéneas son sectores de la ciudad que presentan características internas similares en cuanto al comportamiento de una serie de variables socio-demográficas y energéticas predefinidas. Para su delimitación se necesita, inicialmente, caracterizar a las unidades censales más pequeñas [radios censales (RC)] y, luego, agruparlos según su afinidad.

Por lo tanto, primero, es necesario representar territorialmente una serie de variables (a) socio-demográficas y (b) energéticas para reconocer de manera separada su comportamiento, detectar regiones con características particulares y, a su vez, detectar correlaciones entre las variables. Para ello, se elaboran mapas con las variables que se exponen a continuación:

(a) Variables censales socio-demográficas (geo-referenciadas por RC): 1Personas/hogar; 2- Habitaciones/hogar; 3- [\%] Hogares con necesidades básicas insatisfechas; 4- [\%] Hogares con gas por red; 5- [\%] Hogares con tipología de vivienda 'Casa'; 6- [\%] Hogares con vivienda de calidad constructiva 'Satisfactoria'; 7Habitantes/hectárea; 8- Hogares/hectárea; 9- Personas ocupadas; 10- Edad promedio y 11[\%] Hogares con vivienda propia.

(b) Variables energéticas (geo-referenciadas por RC): 1.- Demanda eléctrica promedio por usuario; 2.- Demanda de gas natural por red promedio por usuario y 3.- Demanda de combustibles a granel promedio por usuario.

Para analizar sus niveles de correlación, y utilizando como unidad de análisis a los radios censales, se elaboró una ficha temática para cada una de las once variables socio-demográficas 
donde se construyen tres gráficos de dispersión (Figura 2). Cada gráfico representa el cruce entre la variable socio-demográfica y las tres variables energéticas. De allí se obtienen los grados de correlación $\left(\mathrm{R}^{2}\right)$ de cada una de las variables consideradas.

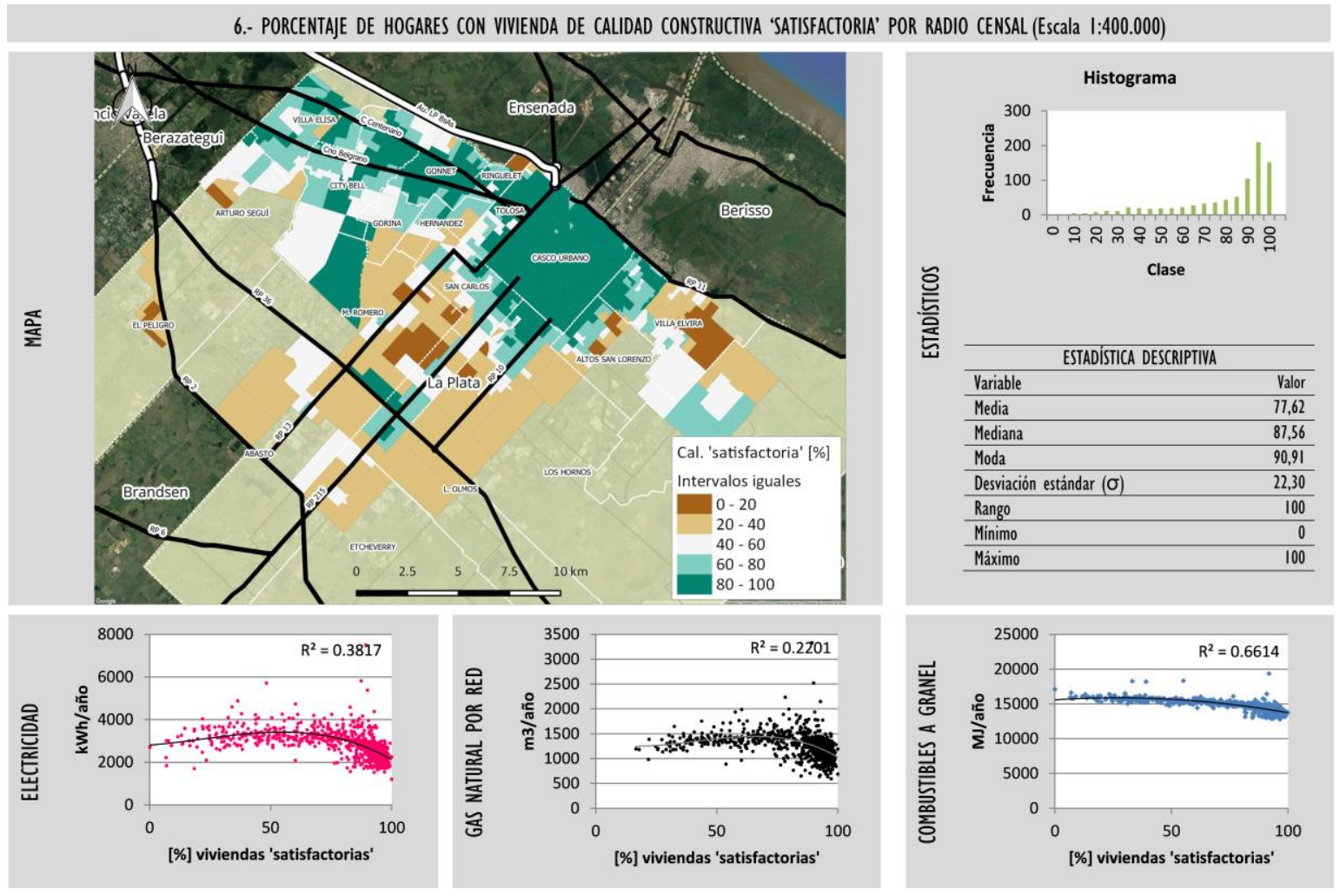

Figura 2 - Ficha temática de una de las variables socio-demográficas y el cruce con la demanda energética. Fuente: elaboración propia de los autores.

A partir de reconocer aquellos cruces con mayor valor de $\mathrm{R}^{2}$, se seleccionan cuatro variables socio-demográficas y dos variables energéticas, las cuales permitirán realizar el agrupamiento urbano-energético de radios censales $(\mathrm{RC})$ para delimitar las áreas homogéneas $(\mathrm{AH})$ : en este caso las variables utilizadas fueron: Personas/hogar; Habitaciones/hogar; Hogares con tipología de vivienda 'casa' [\%]; Hogares/ha.; Consumo promedio de electricidad [kWh/año por usuario]; y Consumo promedio de gas natural [m3/año por usuario].

Para el agrupamiento de los RC, que constituye la segunda etapa, se utiliza la técnica de clustering de k-medias. Las AH obtenidas del agrupamiento de RC presentarán comportamientos internos similares respecto de las seis variables seleccionadas. En este sentido, puesto que el objetivo de delimitar las AH es ensayar la incorporación de sistemas de energía solar teniendo en cuenta los puntos críticos tanto energéticos como sociales y morfológicos, se opta por un agrupamiento que responda a dichos aspectos. En este sentido, para la preparación de los datos, en principio, es necesario asociar la información que se utilizará para el clustering a los 825 radios censales urbanos con los que cuenta el caso de estudio. Por ende, cada RC cuenta con un vector de seis dimensiones que incluye la información urbano-energética seleccionada y cuyos valores deben estar normalizados entre 0 y 1.

En términos operativos, el algoritmo utiliza como insumo " $n$ " cantidad de vectores de " $d$ " dimensiones y debe conocer a priori el número de clases " $k$ " a detectar. Primero, el algoritmo elige al azar del conjunto de " $\mathrm{k}$ " vectores a clasificar, que serán inicialmente los centroides de cada clase (habrá " $k$ " clases). Posteriormente, el algoritmo itera hasta que se encuentra una situación estable que se detalla a continuación. En cada iteración se realizan dos pasos: (i) a cada uno de los "n" 
vectores se le asigna la clase cuya distancia euclídea al centroide sea menor y (ii) se recalculan los centroides de cada clase. Si los centroides de la iteración actual coinciden con los de la anterior iteración, el algoritmo termina (situación estable), en el caso contrario el ciclo continúa realizando los dos pasos anteriores. En este trabajo se tiene: " $n "=825$ (cantidad de radios censales); "d" $=6$ (variables seleccionadas); "k"= 5 (cantidad de grupos a clasificar).

Una vez que se ejecuta el algoritmo, a cada uno de los 825 radios censales se le asigna la pertenencia a un cluster y, a partir de esto, es posible construir un mapa para identificar la ubicación territorial de cada uno de ellos, los cuales constituyen las denominadas áreas homogéneas. Finalmente, se calcula el valor promedio ponderado de distintas variables (V) censales y energéticas en cada una de las AH (Ecuación 1). Dicha ecuación afecta el valor de la variable de cada RC con el peso relativo del mismo sobre el AH y, para ello, se utiliza el cociente entre la cantidad de hogares del RC y la cantidad total de hogares en el AH. La expresión se observa a continuación:

Donde

$$
\bar{X}_{v}=\sum_{i=1}^{n} V_{i} *\left(\frac{\operatorname{Hog}_{i}}{\operatorname{Hog}_{t o t}}\right)
$$

$-\overline{\mathrm{X}}_{\mathrm{V}}=$ promedio ponderado de la variable $\mathrm{V}$ en un área homogénea

$-\mathrm{V}_{\mathrm{i}}=$ valor de la variable en el radio censal (i)

- $\mathrm{Hog}_{\mathrm{i}}=$ cantidad de hogares en el radio censal (i)

- Hog tot $=$ cantidad total de hogares en el área homogénea

Por su parte, siguiendo la metodología planteada en Chévez et al. (2016), es posible identificar el nivel de consolidación urbana de cada uno de los RC que conforman el territorio (alta; media y baja). Para ello se analiza la intensidad de ocupación del territorio a partir de la densidad poblacional, edilicia y la cantidad de redes de servicios disponibles. Posteriormente, para cada $\mathrm{AH}$, se contabilizan los RC pertenecientes a cada nivel de consolidación y se determina una categorización global para cada una de ellas (alta; media-alta; media; media-baja y baja).

Una vez analizadas las $\mathrm{AH}$, es posible relevar información detallada de las distintas variables involucradas a partir del análisis de los MU.

\subsection{Métodos para la selección, caracterización morfológica, termo-física y climática de mosaicos urbanos}

Para realizar el estudio morfológico, termo-físico y climático de los distintos sectores de la ciudad se plantea la utilización de mosaicos urbanos; los cuales son pequeñas porciones de la ciudad. Los MU son representativos de áreas mayores que, por consiguiente, permiten el estudio de las mismas sin necesidad de efectuar un relevamiento sobre toda su extensión (Viegas, 2010). En este trabajo, se adoptará el estudio de un MU por cada AH delimitada.

Para obtener información sobre las características morfológicas y termo-físicas de cada MU, se realiza la observación de imágenes aéreas y satelitales, como así también relevamientos in situ. Este procedimiento consiste en relevar el área y representar los datos obtenidos con programas $\mathrm{CAD}$, para obtener gráficos simplificados (2D y 3D) de los llenos/vacíos y de la volumetría real. A partir de éstos, se obtiene una cuantificación de las superficies expuestas, las cuales permiten calcular otros indicadores de interés como el coeficiente volumétrico de pérdidas de calor $\left[\mathrm{G}_{\mathrm{cal}}\right]$, el asoleamiento de muros y techos, entre otros. A su vez, en cada MU se realizan mediciones de temperatura exterior para el mes de junio/julio con micro adquisidores de datos del tipo Hobo.

En consecuencia, se selecciona un MU de cada área homogénea, teniendo en cuenta su representatividad estadística en el área y considerando su disponibilidad para instalar el equipamiento de medición. Las categorías de variables analizadas para cada uno de los mosaicos se exponen a continuación: 
Inicialmente se estudia la [1] Representatividad del MU. Allí se analizan los estadísticos que muestran la distancia del vector del RC donde se ubica el MU seleccionado, respecto del vector centroide del AH obtenido a partir del clustering. Con el histograma y la distancia euclídea se observa en qué medida es representativo el MU seleccionado.

Posteriormente, se analizan [2] Características morfológicas, donde se obtienen de forma desagregada las superficies que conforman el MU. Aquí se identifican, por ejemplo, superficies de muros orientados al NO/NE y techos, las cuales interesan directamente para el cálculo del potencial de inserción de sistemas de energía solar. También se evalúan las obstrucciones solares de los muros NO/NE y de techos, para la situación más desfavorable (21 de junio), utilizando el modelo gráfico computacional desarrollado por Mesa et al. (1999) que es una rutina que funciona bajo un entorno CAD.

También se estudia la [3] Calidad constructiva, donde se relevan los datos de la calidad de los materiales que componen la envolvente de muros, pisos y aberturas de las viviendas a partir de información del censo para el RC donde se emplaza el MU. Por su parte, la categoría [4] Superficie de techos releva los distintos tipos de tecnologías constructivas de las cubiertas.

En función de las variables e indicadores relevados previamente es posible, en [5] Cálculo térmico, obtener el coeficiente de transmitancia térmica medio $\left(\mathrm{U}_{\text {medio }}\right)$ de muros, ventanas y techos. Para ello, se asignan determinados valores de $\mathrm{U}$ a los distintos elementos constructivos según la calidad de los materiales detectada en [3]. A partir del cálculo del U de los elementos, se calcula el coeficiente de transmitancia térmica medio del $\mathrm{MU}\left(\mathrm{U}_{\text {medio }} \mathrm{MU}\right)$ utilizando como simplificación que un $15 \%$ de la superficie vertical es ocupada por ventanas.

Posteriormente, se calcula el [6] Coeficiente volumétrico de pérdidas de calor $\left[\mathrm{G}_{\mathrm{cal}} \mathrm{MU}\right]$ utilizando la Ecuación 2 (IRAM, 2001). También se obtiene valor máximo admisible [ $\mathrm{G}_{\mathrm{adm}} \mathrm{MU}$ ] según la norma IRAM 11604, con el que se puede obtener el ahorro potencial de energía para calefacción en el mosaico estudiado.

Donde

$$
G_{c a l} M U\left[W / m^{3} * K\right]=\frac{U_{\text {medio }} M U\left[W / m^{2} * K\right] * S_{\text {env }}\left[m^{2}\right]}{V\left[m^{3}\right]}+(0,35 * n)
$$

- $\mathrm{G}_{\text {cal }} \mathrm{MU}=$ coeficiente volumétrico de pérdidas de calor

- $\mathrm{U}_{\text {medio }} \mathrm{MU}=$ es el coeficiente de transmitancia promedio de todo el mosaico

$-\mathrm{S}_{\text {env }}=$ es la superficie total expuesta de la envolvente

- V= es el volumen edificado del mosaico en metros cúbicos

- 0,35= capacidad específica asumida del aire, en vatios hora por $\mathrm{m}^{3}$ por grados kelvin

- $\mathrm{n}=$ es el número de renovaciones de aire promedio por hora y se adopta un valor de $\mathrm{n}=2$

Por último, a partir de mediciones de temperaturas externas tomadas en los MU, se cuenta con la [7] Condición climática de cada uno de estos. Utilizando mediciones para el mes de junio/julio, se calcularon los Grados día de calefacción en base $18^{\circ} \mathrm{C}$ (Viegas et al., 2017), cuya utilidad radica en conocer la incidencia del ambiente construido sobre el clima urbano y, de esta manera, contar con mayores herramientas para evaluar el impacto de medidas de mejoramiento energético y la fracción solar del consumo de calefacción que es posible cubrir en las distintas AH.

\subsection{Método para determinar el potencial solar de los diferentes MU asociados al AH}

A partir de la información relevada es posible caracterizar las diferentes áreas homogéneas de la ciudad $\mathrm{y}$, en función de ello, identificar la potencialidad o las dificultades para la implementación de las diferentes tecnologías de energía solar. Cómo se explicitó previamente, éstas dependen de las características físicas, morfológicas, constructivas y térmicas externas de cada $\mathrm{AH}$, dado que están estrechamente ligadas a las superficies disponibles asoleadas y de las obstrucciones solares. A su vez, es necesario considerar las condiciones socio-demográficas o económicas, 
atendiendo a factores como la proporción de inquilinos/propietarios, niveles de NBI, presencia del gas por red, entre otros factores. Estos inciden directamente en la viabilidad de aplicación los distintos sistemas solares, tales como los sistemas pasivos para calefacción (muros acumuladores, invernaderos, colectores de aire y ganancia directa); los colectores solares de agua caliente sanitaria o los paneles fotovoltaicos (FV) para generación eléctrica.

En consecuencia, para obtener el índice de potencial solar máximo de cada área, primero, se calcula la fracción solar alcanzada por la implementación de sistemas pasivos solares para calefacción en muros (Fsp). Luego, se obtiene la fracción solar resultado de la instalación de colectores solares para la producción de agua caliente (Facs) y para sistemas FV (Ffv), ambos en techos. Finalmente, se obtiene un índice que integra los valores de muros y techos, el cual permite caracterizar rápidamente cada $\mathrm{AH}$. Allí solamente se considerará viable la implementadas de sistemas solares únicamente en aquellas viviendas que estén habitadas por sus propietarios, y no en aquellas que son de alquiler. Para ello, sobre cada uno de los MU se aplica la siguiente metodología:

\subsubsection{Obtención del indicador de potencial solar máximo para sistemas pasivos en muros}

Para obtener el indicador del potencial solar máximo en muros, en primer lugar, se calcula el aporte de un sistema pasivo genérico aplicado sobre la superficie vertical disponible (NE/NO) en una vivienda promedio del MU para el mes más desfavorable -junio- (Ecuación 3).

Donde

$$
Q s p_{i}[k W h]=A_{p}\left[m^{2}\right] * H\left[M J / m^{2} * \text { día }\right] * 30[\text { días }] * \eta_{s p} * 0,277[k W h / M J]
$$

- $\mathrm{Qsp}_{\mathrm{i}}=$ aporte de energía a una vivienda promedio del MU por parte de un sistema pasivo i $(\mathrm{kWh})$

- $\mathrm{A}_{\mathrm{p}}=$ es la superficie de muros disponible $\mathrm{NE} / \mathrm{NO}$ de una vivienda promedio en el MU $\left(\mathrm{m}^{2}\right)$

- $\mathrm{H}=$ promedio diario de radiación solar sobre un plano a $90^{\circ}$ orientado al NO/NE en junio $\left(8,4 \mathrm{MJ} / \mathrm{m}^{2} *\right.$ día $)$

- 30 días = días del mes de junio

- $\eta_{s p}=$ eficiencia de un sistema pasivo genérico. Promedio entre muros acumuladores (30\%), invernaderos $(40 \%)$, colectores solares de aire (50\%) y ganancia directa (70\%), relevado en Chévez (2017). Se adopta un valor medio del $50 \%$.

Asimismo, se calcula la carga térmica del MU para dicho mes (Ecuación 4), utilizando las expresiones de la norma IRAM 11604 (IRAM, 2001):

Donde

$$
Q_{c a l}[k W h]=\frac{24 * G_{m e d}\left[W / m^{3} K\right] * \operatorname{Vol}_{v}\left[m^{3}\right] * G D_{j u n}}{1000}
$$

- $\mathrm{Q}_{\mathrm{cal}}=$ demanda teórica de calefacción de una vivienda promedio del MU para junio $(\mathrm{kWh})$

- 24= tiempo de calefacción en horas por día

- $\mathrm{G}_{\mathrm{med}}=$ coeficiente volumétrico de pérdidas del $\mathrm{MU}\left(\mathrm{W} / \mathrm{m}^{3} \mathrm{~K}\right)$

- $\mathrm{Vol}_{\mathrm{v}}=$ volumen de una vivienda promedio del MU

- $\mathrm{GD}_{\text {jun }}=$ grados día calculados a partir de mediciones de $\mathrm{T}^{\mathrm{o}}$ en el MU

La Ecuación 5 calcula la fracción solar que es posible cubrir con la superficie de muros (NE/NO) asoleados:

Donde

$$
F s p=Q s p / Q c a l
$$

- Fsp= fracción solar del sistema pasivo de calefacción

- Qsp= aporte de energía a la vivienda promedio del MU por parte del sistema

- Qcal= demanda teórica de una vivienda promedio del MU

\subsubsection{Obtención de indicadores de potencial solar máximo para sistemas activos en techos}

Para obtener los indicadores de potencial solar en techos es necesario considerar que de la superficie de cubiertas disponible, inicialmente se ocupará el área necesaria para abastecer la 
demanda de agua caliente con colectores solares; mientras que la superficie remanente se destinará para la incorporación de paneles FV para la generación eléctrica.

En consecuencia, para el cálculo de la superficie de techos necesaria para los colectores solares de agua se aplica la Ecuación 6.

$$
S T_{\text {csol }}\left[m^{2} \text { techo } / \text { viv }\right]=\left(\frac{P[\text { Pers } / \text { viv }] * 50[\text { lts } / \text { Pers }]}{\eta_{\text {csol }}\left[\text { lts } / m^{2} \text { csol }\right]}\right) * 3,5\left[m^{2} \text { techo } / m^{2} \text { csol }\right]
$$

Donde

- $\mathrm{ST}_{\text {csol }}=$ Superficie de techos destinada a colectores solares de agua promedio por vivienda en el MU

- $\mathrm{P}=$ cantidad de personas por hogar en el $\mathrm{AH}$

- 50= estimación de requerimiento de agua caliente por persona en la ciudad de La Plata

- $\eta_{\text {csol }}=$ rendimiento del colector: relación entre el volumen a acumular y la superficie de la placa absorbedora necesaria. Se adopta un valor de 75 lts de agua caliente por cada $\mathrm{m}^{2}$ de colector solar

- 3,5 es la superficie horizontal de techos necesaria por cada $\mathrm{m}^{2}$ de colector solar instalado (Viegas, 2010)

Con la estimación propuesta, según Lanson et al. (2013), se lograría cubrir un 65\% de la demanda de agua caliente sanitaria debido a la presencia de períodos con baja heliofanía, lo cual resulta en la siguiente fracción solar (Ecuación 7):

Donde

$$
\text { Fcsol }=0,65
$$

- Fcsol= fracción solar para colectores solares de agua caliente en una vivienda promedio del MU

- 0,65= valor fijo de la fracción solar que contempla períodos de baja heliofanía

Para calcular el potencial de producción de energía eléctrica FV, a la superficie de techos promedio por vivienda de cada MU se le resta la superficie calculada necesaria para los colectores solares de agua, y se lo multiplica por la producción media por $\mathrm{m}^{2}$ de un sistema tipo (Ecuación 8):

$$
E_{f v}[k W h / a n ̃ o]=\left(\frac{\left(\left(S T_{t o t} * F_{o b j}\right)-S T_{c s o l}\right)}{3,5\left[m^{2} t e c h o / m^{2} f v\right]}\right) * 0,35\left[k W h / d i ́ a * m^{2} f v\right] * 365[\text { días } / a n ̃ o]
$$

Donde

- $\mathrm{E}_{\mathrm{fv}}=$ potencial máximo de energía eléctrica generada por paneles fv en una vivienda promedio del MU

$-\mathrm{ST}_{\mathrm{tot}}=$ superficie de techos total promedio por vivienda en el MU

- $\mathrm{F}_{\mathrm{obj}}=$ factor que considera una disminución en la superficie de techos por la presencia de elementos tales como antenas, tanques, entre otros. Se utiliza un factor de 0,85

$-\mathrm{ST}_{\text {csol }}=$ Superficie de techos destinada a colectores solares de agua promedio por vivienda en el MU

- 3,5 es la superficie horizontal de techos necesaria por cada $\mathrm{m}^{2}$ de colector solar instalado (Viegas, 2010)

- 0,35 es la producción media diaria de electricidad de un $\mathrm{m}^{2}$ de un sistema FV en La Plata (Chévez, 2017)

Una vez obtenida la producción máxima de electricidad FV, se obtiene el la fracción solar que es posible cubrir con estas tecnologías por medio de la Ecuación 9:

Donde

$$
F f v=E_{f v} / E_{t o t}
$$

- Ffv = fracción solar del sistema fotovoltaico

- $\mathrm{E}_{\mathrm{fv}}=$ potencial máximo de energía eléctrica generada por paneles fv en una vivienda promedio del MU

- $\mathrm{E}_{\mathrm{tot}}=$ es el promedio de consumo de energía eléctrica por vivienda en el $\mathrm{AH}$

\subsubsection{Cálculo del índice integrador del potencial solar máximo de muros y techos}

Una vez obtenidas las fracciones solares de los sistemas pasivos de calefacción (Fsp), colectores de agua caliente (Fcsol) y sistemas FV (Ffv), es necesario integrar a todas ellas en una única expresión que considere la incidencia de dichos sistemas sobre la demanda de gas natural, combustibles a granel y electricidad (Ecuación 10). Para ello, se utiliza la incidencia de cada vector sobre la energía neta de cada $\mathrm{AH}$, la cual se releva en Tabla 1. A su vez, se requiere considerar la incidencia de las fracciones solares sobre la participación de sus correspondientes usos dentro de la 
demanda total de cada vector. Para ello, se adoptan valores fijos obtenidos de Chévez (2017) para la ciudad de La Plata.

En lo que refiere al consumo de gas natural, la participación de los usos es la siguiente: calefacción ( Pcal $\left._{\text {gas }}=53 \%\right)$ y agua caliente sanitara $\left(\right.$ Pacs $\left._{\text {gas }}=30 \%\right)$. En cuanto a los combustibles a granel la distribución adoptada es: calefacción $\left(\mathrm{Pcal}_{\mathrm{gr}}=19 \%\right)$ y agua caliente sanitaria $\left(\mathrm{Pacs}_{\mathrm{gr}}=23 \%\right)$. Mientras que dentro de los consumos eléctricos se utiliza: calefacción ( $\left.\mathrm{Pcal}_{\mathrm{e}}=12 \%\right)$ y agua caliente sanitaria $\left(\mathrm{Pacs}_{\mathrm{e}}=3 \%\right)$; donde a las fracciones individuales también es preciso adicionar la Ffv que cubriría todos los usos de este vector.

Finalmente, se requiere afectar el valor obtenido con la proporción de viviendas que efectivamente podría incorporar sistemas solares (proporción de propietarios). De esta manera, se obtiene el índice integrador del potencial solar para el AH, tal como se observa en la Ecuación 10:

$$
\begin{aligned}
& I p s=\left\{\left[\left[\left(F s p * \text { Pcal }_{\text {gas }}\right)+\left(\text { Fcsol } * \text { Pacs }_{\text {gas }}\right)\right] * G N\right]\right. \\
+ & {\left[\left[\left(F s p * \text { Pcal }_{\text {gr }}\right)+\left(\text { Fcsol } * \text { Pacs }_{g r}\right)\right] * C G\right] } \\
+ & {\left.\left[\left[\left(F s p * \text { Pcal }_{e}\right)+\left(\text { Fcsol } * \text { Pacs }_{e}\right)+(F f v)\right] * E\right]\right\} * P_{A H} }
\end{aligned}
$$

Donde

- Ips= índice integrador de potencial solar para el AH

- Fsp= fracción solar del sistema pasivo de calefacción en el AH

- Fcsol=fracción solar del sistema solar de agua caliente sanitaria en el AH

- Ffv= fracción solar del sistema fotovoltaico en el AH

- Pcal $_{\mathrm{i}}=$ participación del uso "calefacción" dentro del consumo de i=gas (gas natural); i=gr (combustibles a granel); i=e (electricidad). Pcal ${ }_{\mathrm{gas}}=0,53 ; \mathrm{Pcal}_{\mathrm{gr}}=0,19 ; \mathrm{Pcal}_{\mathrm{e}}=0,12$. (Chévez, 2017).

- Pacs $_{\mathrm{i}}=$ participación del uso "agua caliente sanitaria" en el consumo de $\mathrm{i}=$ gas (gas natural); $\mathrm{i}=\mathrm{gr}$ (combustibles a granel); $\mathrm{i}=\mathrm{e}$ (electricidad). $\mathrm{Pacs}_{\mathrm{gas}}=0,53 ; \mathrm{Pacs}_{\mathrm{gr}}=0,23 ; \mathrm{Pacs}_{\mathrm{e}}=0,03$. (Chévez, 2017).

- $\mathrm{GN}=$ incidencia del vector gas natural sobre el consumo total en el AH (Tabla 1)

- $\mathrm{CG}=$ incidencia del vector combustibles a granel sobre el consumo total en el AH (Tabla 1 )

- E= incidencia del vector electricidad sobre el consumo total en el AH (Tabla 1)

- $\mathrm{P}_{\mathrm{AH}}=$ porcentaje de propietarios de vivienda en el $\mathrm{AH}$

\section{RESULTADOS: LA CIUDAD DE LA PLATA, DELIMITACIÓN DE CINCO ÁREAS HOMOGÉNEAS Y SELECCIÓN DE MOSAICOS URBANOS REPRESENTATIVOS.}

En función de la metodología propuesta, se presentan los resultados: (i) descripción de las AH urbano-energéticas; (ii) descripción de los MU; y (iii) análisis de la potencialidad de incorporación de sistemas de energía solar.

\subsection{Delimitación y caracterización de cinco AH residenciales para la ciudad de La Plata}

Se cuenta con los valores asociados de las variables seleccionadas para cada uno de los 825 $\mathrm{RC}$ que conforman el área de estudio, normalizados entre 0 y 1 . A partir de éstos, se realiza su agrupamiento con la técnica de clustering de k-medias utilizando el software SPSS. Dado que este algoritmo necesita conocer a priori el número de clusters $(\mathrm{k})$ a clasificar, se realizaron diferentes pruebas de agrupamientos, partiendo desde cuatro hasta ocho clusters $(4 \leq \mathrm{k} \leq 8)$. A partir de los análisis realizados se optó por una clasificación de k=5.

El resultado territorial se aprecia en la Figura 3, donde se observan los cinco clusters de radios censales obtenidos que conforman las respectivas áreas homogéneas ( $\mathrm{AH})$. Allí se detecta, en primera instancia, un AH localizada en el centro del trazado fundacional, es decir, el casco urbano, la cual se denomina AH1-Casco centro; luego, a su alrededor, una segunda AH que se extiende hasta los límites del casco urbano denominada AH2-Casco bordes; posteriormente, se observa un AH que rodea el trazado fundacional y que, también, abarca sectores intersticiales de la periferia, llamada AH3- Expansión del casco; por otro lado, en el eje noroeste, conformado por las localidades de Gonnet, City Bell y parte de Villa Elisa y M. Romero, se ubica la denominada AH4- 
Eje noroeste y, en última instancia, se observa un $\mathrm{AH}$ que cubre los radios censales periféricos de menor consolidación, llamada AH5-Periurbano.

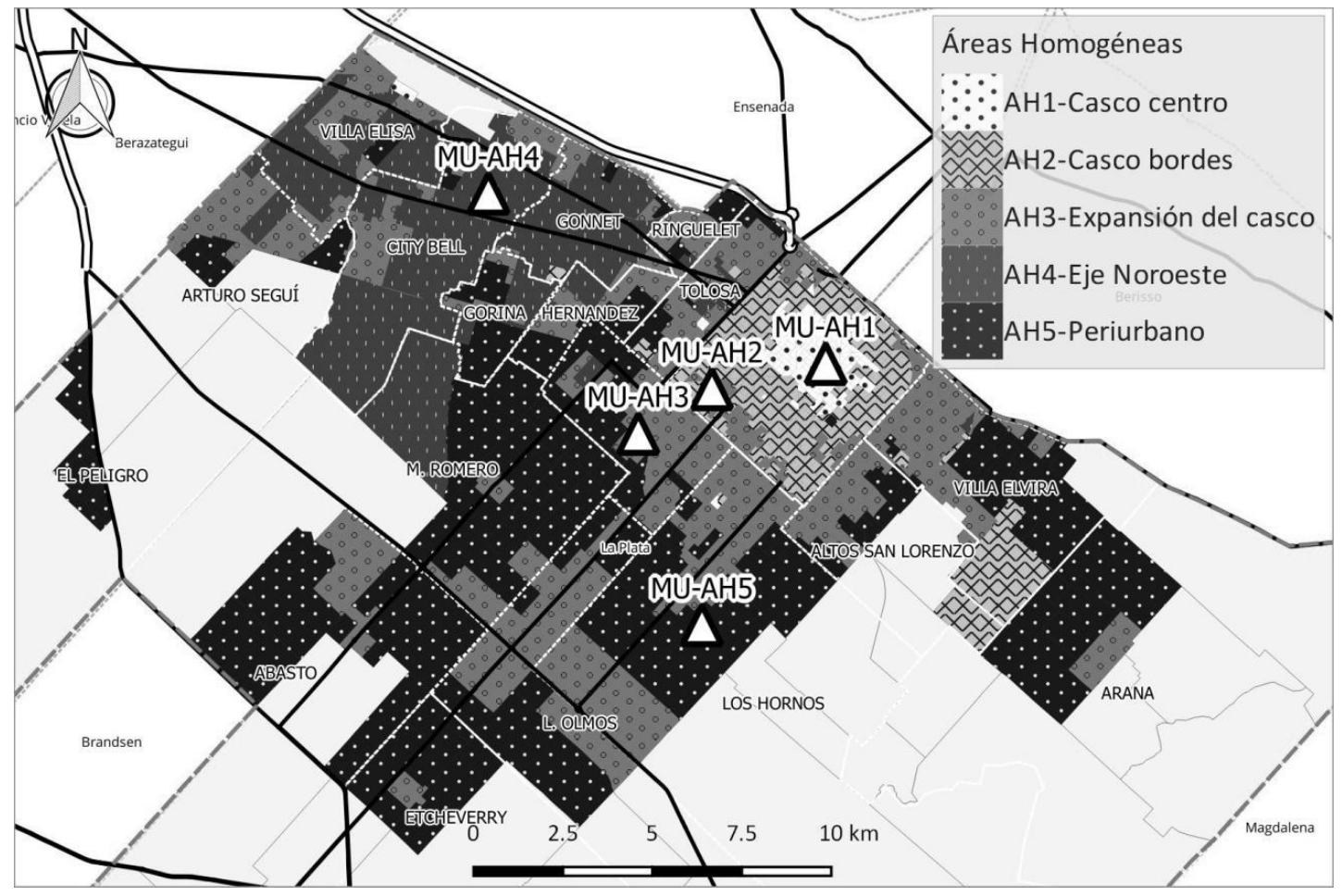

Figura 3 - Resultado territorializado de la clasificación de las AH. Se señala la ubicación de los MU relevados.

Fuente: elaboración propia de los autores.

Una vez obtenidas las $\mathrm{AH}$, es posible describirlas a partir de diferentes variables presentes en el censo, información energética geo-referenciada o por medio de análisis más detallados, como el relevamiento de mosaicos urbanos. En este sentido, se analizan diferentes variables que sirven como insumo para la descripción de cada una de las $\mathrm{AH}$, lo cual es de utilidad al momento de evaluar la implementación de sistemas no convencionales. La Tabla1 resume las variables sociodemográficas y energéticas estudiadas.

Tabla 1 - Representatividad de los cinco mosaicos urbanos asociados a cada una de las AH

\begin{tabular}{|l|r|r|r|r|r|}
\hline \multicolumn{1}{|c|}{ Variable } & \multicolumn{1}{c|}{ AH1 } & \multicolumn{1}{c|}{ AH2 } & \multicolumn{1}{c|}{ AH3 } & \multicolumn{1}{c|}{ AH4 } & AH5 \\
\hline Cantidad de radios censales & 132 & 202 & 292 & 84 & 115 \\
\hline Habitantes & 62080 & 130779 & 266361 & 70224 & 136594 \\
\hline Hogares & 32041 & 56182 & 90165 & 23628 & 37555 \\
\hline Superficie (km ${ }^{2}$ ) & 5,35 & 26,75 & 88,84 & 54,37 & 121,34 \\
\hline Habitantes/ha & 116,09 & 48,89 & 29,98 & 12,92 & 11,26 \\
\hline Hogares/ha & 59,92 & 21,00 & 10,15 & 4,35 & 3,09 \\
\hline Personas/hogar & 1,94 & 2,33 & 2,95 & 2,97 & 3,64 \\
\hline Habitaciones/hogar (para cualquier uso) & 2,86 & 3,33 & 3,26 & 3,93 & 2,64 \\
\hline [\%] Personas ocupadas & 60,45 & 65,64 & 65,41 & 68,54 & 68,42 \\
\hline$[\%]$ Vivienda propia & 46,78 & 63,29 & 77,47 & 83,66 & 82,56 \\
\hline$[\%]$ NBI & 2,25 & 1,48 & 6,60 & 3,00 & 28,40 \\
\hline$[\%]$ Gas por red & 98,90 & 97,45 & 78,09 & 85,37 & 24,43 \\
\hline [\%] & 17,39 & 53,67 & 86,84 & 96,04 & 74,47 \\
\hline Tipologías & 82,56 & 46,06 & 8,99 & 2,19 & 1,98 \\
\hline
\end{tabular}




\begin{tabular}{|c|c|c|c|c|c|c|}
\hline & Viv. precaria & 0,04 & 0,27 & 4,16 & 1,77 & 23,55 \\
\hline \multirow{3}{*}{$\begin{array}{l}{[\%] \text { Calidad }} \\
\text { constructiva }\end{array}$} & Satisfactoria & 93,60 & 92,65 & 75,21 & 80,48 & 34,35 \\
\hline & Básica & 5,63 & 6,45 & 16,63 & 13,98 & 28,38 \\
\hline & Insuficiente & 0,77 & 0,90 & 8,16 & 5,54 & 37,27 \\
\hline \multirow{3}{*}{$\begin{array}{l}\text { Consumo } \\
\text { promedio por } \\
\text { vivienda }\end{array}$} & Electricidad (kWh/año) & 2021,2 & 2451,4 & 3050,9 & 3742,2 & 3186,08 \\
\hline & Gas por red (m³/año) & 945,3 & 1199,3 & 1359,6 & 1654,2 & 1239,50 \\
\hline & Comb. a granel (MJ/año) & 13659 & 14378 & 15533 & 15566 & 16790 \\
\hline \multirow{3}{*}{$\begin{array}{l}\text { Incidencia } \\
\text { sobre el } \\
\text { consumo total }\end{array}$} & [E] Electricidad (\%) & $17 \%$ & $16 \%$ & $20 \%$ & $19 \%$ & $32 \%$ \\
\hline & {$[\mathrm{GN}]$ Gas por red $(\%)$} & $83 \%$ & $83 \%$ & $74 \%$ & $78 \%$ & $33 \%$ \\
\hline & [CG] Comb. granel (\%) & $0 \%$ & $1 \%$ & $6 \%$ & $3 \%$ & $35 \%$ \\
\hline \multicolumn{2}{|c|}{ Nivel de consolidación } & Alta & Media-alta & Media-baja & Baja & Baja \\
\hline
\end{tabular}

Fuente: Elaborado por los autores (2018).

\subsection{Selección y caracterización de mosaicos urbanos para la ciudad de La Plata}

A partir de la selección de un MU por AH, es preciso verificar la representatividad del MU seleccionado en cada AH. Para ello, se recurre a la información utilizada para la ejecución del clustering y se analiza, para cada uno de los cinco casos, la distancia euclidiana simple entre el centroide del RC sobre el cual se emplaza el MU y el centroide de su correspondiente cluster (AH). Asimismo, para cada caso, se construye un histograma que permite evaluar la frecuencia de aparición del rango al que pertenece la distancia obtenida (Tabla 2). En los cinco casos se obtienen distancias que se ubican en rangos que tienen una alta frecuencia de aparición, por lo tanto es posible afirmar que presentan un alto grado de representatividad del $\mathrm{AH}$ a la que pertenecen.

Tabla 2 - Representatividad de los cinco mosaicos urbanos asociados a cada una de las AH

\begin{tabular}{|c|c|c|c|c|c|}
\hline $\begin{array}{c}\text { Representativi } \\
\text { dad }\end{array}$ & AH1-CascoEje & AH2- La Loma & $\begin{array}{c}\text { AH3-San } \\
\text { Carlos }\end{array}$ & $\begin{array}{c}\text { AH4-City Bel } \\
\text { centro }\end{array}$ & $\begin{array}{c}\text { AH5-Los } \\
\text { Hornos }\end{array}$ \\
\hline \multicolumn{6}{|l|}{ Imagen aérea } \\
\hline $\begin{array}{l}\text { Histograma de } \\
\text { frecuencia de } \\
\text { distancias de los } \\
\text { RC }\end{array}$ & 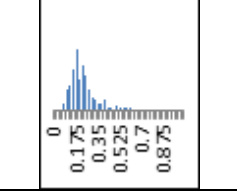 & 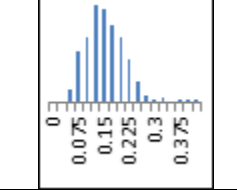 & 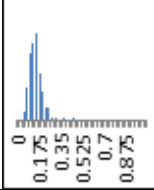 & 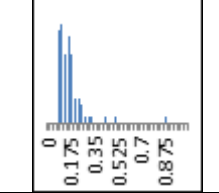 & 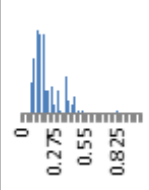 \\
\hline Dist. Euclídea & 0,25 & 0,13 & 0,08 & 0,069 & 0,37 \\
\hline
\end{tabular}

Fuente: Elaborado por los autores (2017).

Por su parte, en Tabla 3 se relevan el resto de las variables propuestas en la sección 4.2.

Tabla 3- Relevamiento de los cinco mosaicos urbanos asociados a cada una de las AH.

\begin{tabular}{|l|c|c|c|c|c|}
\hline Características morfológicas & MU1-AH1 & MU1-AH2 & MU3-AH3 & MU4-AH4 & MU5-AH5 \\
\hline Superficie de terreno $\left(\mathrm{m}^{2}\right)$ & 14518 & 14690 & 14335 & 9927 & 7937 \\
\hline Envolvente vertical $\left(\mathrm{m}^{2}\right)$ & 23421 & 8352 & 7299 & 4056 & 2151 \\
\hline Muros $\left(\mathrm{m}^{2}\right)$ & 19908 & 7099 & 6204 & 3448 & 1828 \\
\hline Ventanas $\left(\mathrm{m}^{2}\right)$ & 3513 & 1253 & 1095 & 608 & 323 \\
\hline Muros NO $\left(\mathrm{m}^{2}\right)$ & 3875 & 1364 & 1408 & 673 & 564 \\
\hline Muros NE $\left(\mathrm{m}^{2}\right)$ & 2783 & 1182 & 1197 & 958 & 478 \\
\hline Techos total superficie $\left(\mathrm{m}^{2}\right)$ & 9887 & 5951 & 4616 & 3262 & 1083 \\
\hline Obstrucción solar NO (\%) & $60 \%$ & $43 \%$ & $48 \%$ & $39 \%$ & $29 \%$ \\
\hline Obstrucción solar NE $(\%)$ & $56 \%$ & $22 \%$ & $31 \%$ & $39 \%$ & $14 \%$ \\
\hline Obstrucción solar techo $(\%)$ & $23 \%$ & $10 \%$ & $4 \%$ & $2 \%$ & $1 \%$ \\
\hline Muros NO+NE asoleados $\left(\mathrm{m}^{2}\right)$ & 2774,10 & 1699,44 & 1558,09 & 994,91 & 811,52 \\
\hline Techos asoleados $\left(\mathrm{m}^{2}\right)$ & 7612,99 & 5355,9 & 4431,36 & 3196,76 & 1072,17 \\
\hline Cantidad de viviendas $(\mathrm{Unidad})$ & 137 & 65 & 33 & 23 & 12 \\
\hline$\mu$ Muros NO+NE asoleados $\left[\mathrm{m}^{2} / \mathrm{viv}\right]$ & 20,24 & 26,14 & 47,21 & 43,25 & 67,62 \\
\hline$\mu$ Techos asoleados $\left[\mathrm{m}^{2} / \mathrm{viv}\right]$ & 55,56 & 82,39 & 134,28 & 138,98 & 89,34 \\
\hline
\end{tabular}




\begin{tabular}{|c|c|c|c|c|c|c|c|c|c|c|}
\hline Superficie construida total $\left(\mathrm{m}^{2}\right)$ & \multicolumn{2}{|c|}{17138} & \multicolumn{2}{|c|}{8730} & \multicolumn{2}{|c|}{4965} & \multicolumn{2}{|c|}{2072} & \multicolumn{2}{|c|}{1601} \\
\hline Volumen $\left(\mathrm{m}^{3}\right)$ & \multicolumn{2}{|c|}{109983} & \multicolumn{2}{|c|}{24700} & \multicolumn{2}{|c|}{16369} & \multicolumn{2}{|c|}{9860} & \multicolumn{2}{|c|}{4329} \\
\hline Calidad constructiva & \multicolumn{2}{|c|}{$\%$} & \multicolumn{2}{|c|}{$\%$} & \multicolumn{2}{|c|}{$\%$} & \multicolumn{2}{|c|}{$\%$} & \multicolumn{2}{|c|}{$\%$} \\
\hline Viviendas satisfactorias & \multicolumn{2}{|c|}{93,60} & \multicolumn{2}{|c|}{92,65} & \multicolumn{2}{|c|}{75,21} & \multicolumn{2}{|c|}{80,48} & \multicolumn{2}{|c|}{34,35} \\
\hline Superficie de Techos & $\mathbf{m}^{2}$ & $\%$ & $\mathbf{m}^{2}$ & $\%$ & $\mathbf{m}^{2}$ & $\%$ & $\mathbf{m}^{2}$ & $\%$ & $\mathbf{m}^{2}$ & $\%$ \\
\hline Chapa galvanizada & 7.910 & 80,1 & 4.560 & 76,6 & 4.062 & 87,9 & 1.305 & 40,0 & 791 & 73,0 \\
\hline Teja roja & 0 & 0 & 448 & 7,5 & 369 & 7,9 & 1.957 & 60,0 & 162 & 14,9 \\
\hline Losa & 1.977 & 19,9 & 943 & 15,9 & 185 & 4,2 & 0 & 0 & 130 & 12,1 \\
\hline Cálculo térmico & \multicolumn{2}{|c|}{$\mathbf{U}$} & \multicolumn{2}{|c|}{$\mathbf{U}$} & \multicolumn{2}{|c|}{$\mathbf{U}$} & \multicolumn{2}{|c|}{$\mathbf{U}$} & \multicolumn{2}{|c|}{$\mathbf{U}$} \\
\hline $\mathrm{U}_{\text {medio }}$ muro & \multicolumn{2}{|c|}{2,40} & \multicolumn{2}{|c|}{2,41} & \multicolumn{2}{|c|}{2,50} & \multicolumn{2}{|c|}{2,47} & \multicolumn{2}{|c|}{2,72} \\
\hline $\mathrm{U}_{\text {medio }}$ ventana & \multicolumn{2}{|c|}{4,35} & \multicolumn{2}{|c|}{4,35} & \multicolumn{2}{|c|}{4,48} & \multicolumn{2}{|c|}{4,44} & \multicolumn{2}{|c|}{4,78} \\
\hline $\mathrm{U}_{\text {medio }}$ techo & & & 1 , & & 1 , & & 1, & & & \\
\hline $\mathrm{U}_{\text {medio }} \mathrm{MU}$ & 2 , & & 2, & & 2 , & & 2, & & & \\
\hline Coef. volumétrico de pérdidas & $\mathbf{M}$ & & $\bar{M}$ & & $\mathbf{M}$ & & $\mathbf{M}$ & & & \\
\hline $\mathrm{G}_{\mathrm{cal}} \mathrm{MU}$ & & & 2 , & & 2 , & & 2, & & & \\
\hline $\mathrm{G}_{\mathrm{adm}} \mathrm{MU}$ & & & 1 , & & 1, & & 1 , & & & \\
\hline Condición climática (térmica) & $\bar{M}$ & & $\mathbf{M}$ & & $\mathbf{M}$ & & $\mathbf{M}$ & & & \\
\hline Grados Día $18^{\circ} \mathrm{MU}$ (junio/julio) & 2 & & 13 & & 2 & & 20 & & & \\
\hline
\end{tabular}

Fuente: Elaborado por los autores (2017).

\subsection{Análisis del potencial solar de los MU considerando las variables asociadas del AH}

En función de las superficies promedio disponibles de cada MU y las condiciones sociodemográficas de las $\mathrm{AH}$, se analizan las condiciones que presenta cada una de ellas para la inserción de sistemas solares. Para ello, se calcula la fracción solar para la inserción de sistemas solares pasivos (Fsp); la fracción solar de colectores solares (Fcsol); la fracción solar de sistemas FV (Ffv); y, finalmente, el índice de potencial solar (Ips). Los resultados se observan en la Tabla 4.

Tabla 4- Cálculo de las fracciones solares y el Ips de cada MU

\begin{tabular}{|c|c|c|c|c|c|c|}
\hline & Variable & MU1 & MU2 & MU3 & MU4 & MU5 \\
\hline \multirow{3}{*}{$\sum_{z}^{3}$} & Potencial energético junio $(\mathrm{kWh})$ & 706,70 & 912,28 & 1647,79 & 1508,36 & 2358,79 \\
\hline & Demanda energética junio (kWh) & 5730,80 & 2473,06 & 7649,5 & 5350,12 & 6345,61 \\
\hline & Fracción solar de sist. pasivos de calef. (Fsp) & 0,12 & 0,37 & 0,22 & 0,28 & 0,37 \\
\hline \multirow{4}{*}{$\begin{array}{l}\stackrel{8}{\circlearrowright} \\
\varrho\end{array}$} & Fracción solar de colectores solares (Fcsol) & \multicolumn{5}{|c|}{0,65} \\
\hline & Potencial de generación FV (kWh/año) & 1558,52 & 2357,71 & 3914,79 & 4058,90 & 2461,76 \\
\hline & Demanda eléctrica promedio (kWh/año) & 2021,20 & 2451,40 & 3050,90 & 3742,20 & 3186,08 \\
\hline & Fracción solar FV (Ffv) & 0,77 & 0,96 & 1,28 & 1,08 & 0,77 \\
\hline \multirow{2}{*}{$气$} & Propietarios en el AH & $46,7 \%$ & $63,2 \%$ & $77,4 \%$ & $83,6 \%$ & $82,5 \%$ \\
\hline & Índice de potencial solar (Ips) & 0,16 & 0,31 & 0,39 & 0,41 & 0,38 \\
\hline
\end{tabular}

Fuente: Elaborado por los autores (2017).

A continuación se analizan los resultados obtenidos de potencial solar en cada MU (Tabla 4), integrando información socio-demográfica y energética de su correspondiente AH (Tabla 1).

De los cinco sectores, el MU1 presenta la menor superficie disponible para la instalación de sistemas solares, con valores promedio por vivienda de $20,24 \mathrm{~m}^{2}$ de muros NO/NE y 55,56 $\mathrm{m}^{2}$ de techos, debido a la alta consolidación que presenta el área en la cual se inserta. En la información del AH1 se puede verificar que únicamente un 46,7\% de los hogares es propietario de su vivienda, por lo que la incorporación de sistemas se restringe notablemente. Por su parte, se observa que este sector de la ciudad presenta los hogares más reducidos (1,94 Pers/Hog.), una densidad muy elevada (116,09 Habitantes/Ha.), un nivel de NBI bajo (2,25\%), una presencia del gas por red prácticamente total $(98,90 \%)$, una preponderancia de tipologías de departamentos $(82,56 \%)$ y una calidad constructiva mayormente satisfactoria $(93,6 \%)$. Si bien los hogares son reducidos, la condición térmica externa de invierno no es de las más rigurosas (208 GD) y la condición socio-económica del sector es favorable. En consecuencia, debido a la morfología urbana y a la gran proporción de inquilinos, se obtiene el Ips más bajo de la ciudad $(0,16)$. Por lo tanto, para alcanzar una amplia implementación de sistemas solares, a futuro, se requeriría de una medida particular como por 
ejemplo normativas que soliciten la inserción de estas tecnologías en las edificaciones multifamiliares nuevas, las cuales se destinarán fundamentalmente para alquiler.

El MU2 se inserta en un sector con una consolidación media-alta y, por tal motivo, presenta un promedio por vivienda de $26,14 \mathrm{~m}^{2}$ de muros disponibles orientados al NE/NO y $82,39 \mathrm{~m}^{2}$ de techos. En el $\mathrm{AH} 2$, se genera la transición entre las viviendas con tipología departamento a la tipología casa, más característica de la periferia. En este sentido, un 53,67\% de las viviendas son casas y un $46,06 \%$ son departamentos. Asimismo, la calidad constructiva es principalmente satisfactoria $(92,65 \%)$, la red de gas abarca prácticamente a la totalidad de los hogares $(97,45 \%)$, los niveles de NBI son muy bajos (1,48\%), la densidad desciende significativamente (48,89 Habitantes/Ha.), la media de personas por hogar es de 2,33 y los hogares propietarios de vivienda ascienden a un $63,29 \%$. En síntesis, esta $\mathrm{AH}$ presenta la menor rigurosidad térmica externa de invierno (131 GD), hogares reducidos y una considerable superficie disponible para la incorporación de energía solar en muros $(\mathrm{Fsp}=0,37)$ e intermedia en techos $(\mathrm{Ffv}=0,96)$; $\sin$ embargo, también existe una proporción importante de inquilinos, lo cual genera que el Ips sea el segundo más bajo de la ciudad $(0,31)$. En este sentido, se debería adoptar la misma consideración que en el AH1 referida a las nuevas edificaciones; mientras que para las existentes, teniendo en cuenta que se trata de un sector con buenos indicadores socio-económicos, se podrían implementar incentivos para la difusión de estos sistemas de forma masiva al menos para los propietarios.

El MU3 se inserta en un sector urbano de consolidación media-baja, por lo cual las densidades descienden notablemente, alcanzando los 29,98 Habitantes/Ha. En este sentido, las superficies promedio disponibles de muros orientados al NE/NO son de 47,21 $\mathrm{m}^{2}$ y de techos $134,28 \mathrm{~m}^{2}$, lo cual representa un valor muy elevado. En este sector (AH3), las tipologías más representativas son las casas $(86,84 \%)$, las viviendas con construcción satisfactoria representan un $75,21 \%$, la red de gas logra abastecer a un 78,09\% de los hogares y el nivel de NBI alcanza un valor del 6,6\%. Asimismo, los hogares cuentan con una media de 2,95 personas y la condición de propiedad de la vivienda alcanza a un $77,47 \%$ de los hogares. Las particularidades de este sector indican que las superficies disponibles para equipos solares son vastas, sin embargo la condición térmica externa es muy rigurosa, es por ello que la Fsp no es demasiado elevada (0,22); mientras que el Ffv es muy favorable $(1,28)$, a tal punto que podría generar más energía eléctrica que la demandada. Estas condiciones, junto con la proporción de inquilinos, resultan en un valor de Ips=0,39; que es el segundo más alto de los cinco casos. Por su parte, las condiciones de NBI son más altas que en $\mathrm{AH} 1$ y $\mathrm{AH} 2$, por lo que se necesitarían programas específicos bien concretos y direccionados, como por ejemplo subsidios destinados a la compra de equipamiento no convencional. Cabe destacar que en esta $\mathrm{AH}$, más de un quinto de la población no cuenta con gas por red, por lo que la sustitución de fuentes energéticas costosas como la leña y el GLP por energía solar tendrían un impacto muy positivo en la población.

El MU4 corresponde a un sector de consolidación baja, en el cual la densidad presenta un valor de 12,92 Habitantes/Ha. y, en consecuencia, los valores promedio de muros orientados al NE/NO son de 43,25 $\mathrm{m}^{2}$ y de techos unos $138,98 \mathrm{~m}^{2}$. Las tipologías de vivienda más representativas son las casas, con una participación del $96,04 \%$ y la calidad constructiva es mayormente satisfactoria $(80,48 \%)$. La red de gas abarca a un $85,37 \%$ de los hogares, el nivel de NBI es del $3 \%$ y se encuentra una alta proporción de hogares propietarios de viviendas $(83,66 \%)$. Por su parte, el tamaño de los hogares es de 2,97 Pers./Hog. Esta AH cuenta con las viviendas promedio más grandes de la ciudad, por ende las superficies disponibles para sistemas pasivos son abundantes, con valores de $\mathrm{Fsp}=0,28$ y Ffv=1,08; resultando en el mayor Ips $(0,41)$. A su vez, la condición socioeconómica muestra indicadores satisfactorios, por lo cual se trata de un sector donde los incentivos para la inversión privada podrían ser una política viable. 
El MU5 también se inserta en un sector de consolidación baja (AH5), sin embargo, este presenta condiciones de precariedad considerables. En este sentido, el nivel de NBI del sector alcanza un 28,40\%, presenta una media de 3,64 Pers/Hog. y 2,64 Habitaciones/Hog., lo que representa una condición de hacinamiento importante. La proporción de viviendas con calidad constructiva satisfactoria es del $34,35 \%$, la red de gas alcanza a un $24,43 \%$ de los hogares y un $82,56 \%$ de los hogares son propietarios. Las superficies promedio disponibles de muros orientados al NE/NO son de 67,62 $\mathrm{m}^{2}$ y un promedio de techos de $89,34 \mathrm{~m}^{2}$. En tanto, se obtiene uno de los Fsp más altos, alcanzando un valor de 0,37; mientras que el Ffv alcanza un valor de 0,77; lo cual resulta en un Ips=0,38; lo cual es considerable. Es por ello que en esta $\mathrm{AH}$ se debería focalizar fuertemente en una implementación masiva de sistemas pasivos de calefacción y en la implementación de colectores de agua, relegando los sistemas FV (por su bajo potencial en esta sector y por sus costos). Por su parte, la aplicación de los mencionados sistemas lograría cubrir una cantidad de energía considerable que, en su mayoría, es abastecida por fuentes como la leña, el kerosene o el GLP (debido a la escasa cobertura del gas por red). Además, se trata de un sector con una importante vulnerabilidad social, con indicadores de NBI cercanos al tercio de la población, por lo cual el direccionamiento de medidas debe ser específicamente diseñado y orientado a dicha población, siendo necesario contemplar tanto la instalación de sistemas solares como el mejoramiento de la edilicia existente.

\section{CONCLUSIONES}

El presente trabajo ha permitido analizar la potencialidad de incorporación de sistemas de energía solar en distintos sectores de la ciudad desde una óptica que incluye tanto los aspectos morfológicos-urbanos, termo-físicos y de temperatura de cada sector urbano; como así también la dimensión socio-demográfica, que es de vital importancia para la aplicación de políticas energéticas. En relación a este aspecto, se han observado diferencias sustanciales entre las diferentes áreas homogéneas, lo que conlleva al diseño de medidas diferenciadas y específicas para cada sector, las cuales pueden ser ensayadas mediante la construcción de escenarios en futuras publicaciones.

Por tal motivo, es posible afirmar que la delimitación y el análisis de AH se convierte en un método de utilidad para identificar sectores urbanos con características internas similares pero con diferencias entre sí, puesto que de esta forma es posible obtener una descripción urbano-energética sectorizada de la ciudad. Por su parte, la utilización de MU también ha demostrado ser de utilidad para el estudio morfológico y de asoleamiento de las áreas, dado que no se requiere del relevamiento de la totalidad del área mediante técnicas extensivas y costosas, como puede ser la tecnología LiDAR. En última instancia, la combinación de estas dos aproximaciones ha permitido profundizar en el estudio de los sectores urbanos, logrando ajustar y validar argumentos para el direccionamiento de las estrategias energéticas.

En cuanto a los resultados obtenidos, en el caso de las áreas de consolidación alta y mediaalta (AH1 y AH2), la baja disponibilidad de superficies y la gran cantidad de viviendas de alquiler dificulta la implementación de los diversos sistemas, ya que se infiere desinterés por parte de los propietarios en realizar mejoras. Por lo tanto, se requeriría focalizar los esfuerzos en regular la calidad constructiva y el diseño e inclusión de sistemas solares en las futuras edificaciones. En relación a los sectores de consolidación media (AH3), se cuenta con condiciones propicias para la adopción de sistemas renovables, sin embargo la condición de propietarios o los niveles de NBI requiere especial atención a la hora del diseño de las políticas a implementar. Por su parte, en los sectores periféricos de baja consolidación (AH4 y AH5) existe un importante potencial para la difusión de los distintos sistemas de energía solar, siendo las condiciones de NBI e ingresos aquellas que pueden colaborar en la identificación de los tipos de programas a implementar. En 
relación a ello, el AH4 sería propicia para la difusión de incentivos, mientras que el AH5 requiere de una intervención más directa tanto en materia de energía solar como en el mejoramiento de la calidad constructiva de la edilicia existente.

A partir de la descripción elaborada previamente, integrando los aspectos específicos y técnicos que definen las posibilidades de incorporar tecnologías solares con la realidad socioeconómica de la población, es posible reafirmar que no es viable proyectar políticas energéticas genéricas para que sean aplicadas a escala urbana, sino que se requiere de un anclaje territorial que permita diagnósticos específicos para identificar las heterogeneidades que presentan las ciudades.

\section{REFERENCIAS}

ABDURAFIKOV, R.; GRAHNA, E.; KANNARI, L.; YPYÄ, J.; KAUKONENC, S; HEIMONENA, I.; PAIHOA, S. An analysis of heating energy scenarios of a Finnish case district. Sustainable Cities and Society, v. 32, pp. 56-66, 2017. DOI: https://doi.org/10.1016/j.scs.2017.03.015

AMADO, M.; POGGI, F.; AMADO, A. Energy efficient city: a model for urban planning. Sustainable Cities and Society, vol. 26, pp. 476-485, 2016. DOI: https://doi.org/10.1016/j.scs.2016.04.011

BRITO, M.; FREITAS, S.; GUIMARÃES, S.; CATITA, C.; REDWEIK, P. The importance of facades for the solar PV potential of a Mediterranean city using LiDAR data. Renewable Energy, vol. 111, pp. 85-94, 2017. DOI: https://doi.org/10.1016/j.renene.2017.03.085

CHÉVEZ, P. Energías renovables y eficiencia energética. Análisis de medidas orientadas al sector residencial. Ciudad Autónoma de Buenos Aires: Diseño Editorial, 2017.

CHÉVEZ, P. Construcción de escenarios urbano-energéticos a partir de la implementación de estrategias de eficiencia energética y energías renovables en el sector residencial. 2018. Tesis de doctorado (Doctorado en Ciencias -Área Energías Renovables-) - Facultad de Ciencias Exactas, Universidad Nacional de Salta, Salta.

CHÉVEZ, P.; BARBERO, D.; MARTINI, I.; DISCOLI, C. Análisis de áreas homogéneas de consumo eléctrico residencial. Identificación de la incidencia de variables sociodemográficas y grados de consolidación. Avances en Energías Renovables y Medio Ambiente-AVERMA-, vol. 20, pp. 1-12, 2016.

COMPAGNON, C. Solar and daylight availability in the urban fabric. Energy and Buildings, vol. 36, pp. 321-328, 2004. DOI: https://doi.org/10.1016/j.enbuild.2004.01.009

DROUILLES, J.; LUFKIN, S.; REY, E. Energy transition potential in peri-urban dwellings: Assessment of theoretical scenarios in the Swiss context. Energy and Buildings, vol. 148, pp. 379-390, 2017. DOI: http://dx.doi.org/10.1016/j.enbuild.2017.05.033

EICKER, U.; NOUVEL, R.; DUMINIL, E.; COORS, V. Assessing passive and active solar energy resources in cities using 3D city models. Energy Procedia, vol. 57, pp. 896 - 905, $2014 . \quad$ DOI: https://doi.org/10.1016/j.egypro.2014.10.299

GADSDEN, S.; RYLATT, M.; LOMAS, K.; ROBINSON, D. Predicting the urban solar fraction: a methodology for energy advisers and planners based on GIS. Energy and Buildings, vol. 35, pp. 37-48, 2003. DOI: https://doi.org/10.1016/S0378-7788(02)00078-6

GHIASSI, N.; MAHDAVI, A. Reductive bottom-up urban energy computing supported by multivariate cluster analysis. Energy and Buildings, vol. 144, pp. 372-386, 2017. DOI: https://doi.org/10.1016/j.enbuild.2017.03.004

GIRARDIN, L.; MARECHAL, F.; DUBUIS, M.; CALAME-DARBELlAY, M.; FAVRAT, D. EnerGis: A geographical information based system for the evaluation of integrated energy conversion systems in urban areas. Energy, vol. 35, pp. 830-840, 2010. DOI: https://doi.org/10.1016/j.energy.2009.08.018

HOWARD, B.; PARSHALl, L.; THOMPSON, J.; HAMMERB, S.; DICKINSOND, J.; MODIA, V. Spatial distribution of urban building energy consumption by end use. Energy and Buildings, vol. 45, pp. 141-151, 2012. DOI: https://doi.org/10.1016/j.enbuild.2011.10.061

IEA. Indicadores de Eficiencia Energética: Bases Esenciales para el Establecimiento de Políticas. París: International Energy Agency, 2015

INDEC. Base de datos REDATAM SP del Censo nacional 2010. Ciudad Autónoma de Buenos Aires: Instituto Nacional de Estadísticas y Censos, 2016. 
IRAM. Norma 11604. Aislamiento térmico de edificios. Verificación de sus condiciones higrotérmicas. Ahorro de energía en calefacción. Ciudad Autónoma de Buenos Aires: Instituto Argentino de Normalización, 2001.

LANSON, A.; RIGHINI, R.; BENITEZ, E.; BEZZO, E.; FILLOY, E.; ROLDÁN, A.; UNGER, H.; IANELLI, L.; GIL, S. Hacia un uso más eficiente del gas. Aprovechamiento de la energía solar en Argentina. En: Encuentro Latinoamericano de Uso Racional y Eficiente de la Energía, 1. 2013, Buenos Aires. Actas...Buenos Aires: Editorial Croquis, 2013.

MARTINI, I. Construcción de escenarios energéticos alternativos de desarrollo urbano: implementación de un modelo de simulación integral orientado al uso eficiente de la energía y sustitución de fuentes por renovables. Proyecto de Investigación Plurianual (PIP) 11220170100185CO -CONICET-. Consejo Nacional de Investigaciones Científicas y Técnicas, 2017.

MESA, A.; DE ROSA, C.; CORTEGOSO, J. Modelo gráfico computacional para la determinación del área de fachadas potencialmente colectoras en medios urbanos. Avances en Energías Renovables y Medio Ambiente, vol. 4, pp. 1.20$1.31,1999$.

MINEM. Balance Energético Nacional 2014. Ciudad Autónoma de Buenos Aires: Ministerio de Energía y Minería, 2017.

MOROSI, J. Ciudad de La Plata: Tres décadas de reflexiones acerca de un singular espacio urbano. La Plata: LINTA-CIC, 1999.

MÖRTBERG, U.; GOLDENBERG, R.; KALANTARI, Z.; KORDAS, O.; DEAL, B.; BALFORS, B.; CVETKOVIC, V. Integrating ecosystem services in the assessment of urban energy trajectories - A study of the Stockholm Region. Energy Policy, vol. 100, pp. 338-349, 2017. DOI: https://doi.org/10.1016/j.enpol.2016.09.031

ONU. World Urbanization Prospects. New York: Organización de las Naciones Unidas, 2014.

POLIS. Identification and mobilization of solar Potentials via local strategies (POLIS), Colonia: Intelligent Energy Europe, 2012.

SVEINBJÖRNSSON, D.; AMER-ALLAM, S.; BAVNHØJ, A.; ALGREN, L.; SCHRØDER PEDERSEN, A. Energy supply modelling of a low-CO2 emitting energy system: Case study of a Danish municipality. Applied Energy, vol. 195, pp. 922-941, 2017. DOI: https://doi.org/10.1016/j.apenergy.2017.03.086

VIEGAS, G.; CHÉVEZ, P.; DISCOLI, C.; SAN JUAN, G. Comportamiento energético de mosaicos urbanos representativos de la ciudad de La Plata en función de las condiciones térmicas externas. En: Encontro Nacional e Latino-Americano de Conforto no Ambiente Construído, 10. 2017, Camboriú. Actas... Porto Alegre: Associação Nacional de Tecnologia no Ambiente Construído, 2017.

VIEGAS, G. (2010). Evaluación del potencial energético e intervenciones de mejoramiento del entorno edilicio en áreas urbanas de media y baja consolidación. 2010. Tesis de doctorado (Doctorado en Ciencias -Área Energías Renovables-) - Facultad de Ciencias Exactas, Universidad Nacional de Salta, Salta.

WEC. Perspective input into the World Energy Council Scenarios: "Innovating Urban Energy". Londres: World Energy Council, 2016.

WEGERTSEDER, P.; LUND, P.; MIKKOLA, J.; GARCÍA ALVARADO, P. Combining solar resource mapping and energy system integration methods for realistic valuation of urban solar energy potential. Solar Energy, vol. 135, pp. 325-336, 2016. DOI: https://doi.org/10.1016/j.solener.2016.05.061

This work is licensed under a Creative Commons Attribution 4.0 International License. 